\title{
René Pedroza Flores* \\ Estudios del futuro para comprender la posibilidad de la universidad ubicua
}

\section{Future studies to understand the possibility of the ubiquitous university}

\begin{abstract}
The future has been explained in a magical, metaphysical, religious and scientific way. In the scientific explanation studies of the future have been developed. These studies have advanced from a predictive conception to the creation of alternative or desirable futures. Studies of the future environment of the university address changes and trends in the short, medium and long term. The future of the university is at stake, rethinking its trend is a necessity in today's society. The relevance of the writing consists in thinking not in philosophizing - the tendency of the university not as something predictive and decisive but as something uncertain because it opens the possibility of constructing different stories with the deconstructionist and critical approach of studies for the future. The thesis is that the university tends to a ubiquitous form, it will be present everywhere at the same time for a multiplicity of digitally connected subjects simultaneously in their uniqueness. The ubiquitous university, it is concluded, will be a brain of augmented intelligence (social intelligence plus artificial intelligence).
\end{abstract}

Keywords | prospective, futurology, deconstruction, post-humanism, artificial intelligence.

Resumen | El futuro se ha explicado de forma mágica, metafísica, religiosa y científica. En la explicación científica se han desarrollado los estudios del futuro. Estos estudios han avanzado desde una concepción predictiva hasta la creación de futuros alternativos o deseables. Los estudios del futuro de la universidad abordan los cambios y la tendencia en el corto, mediano y largo plazo. El futuro de la universidad está en juego, y repensar su tendencia es una necesidad en la sociedad actual. La relevancia del escrito consiste en plantear la tendencia de la universidad no como algo predictivo y determinante sino como algo incierto porque se apertura la posibilidad de construir distintos relatos con el enfoque deconstruccionista y crítico de estudios para el futuro. La tesis es que la universidad tiende a un modelo ubicuo, al hacer-

Recibido: 11 de marzo de 2020.

Aceptado: 3 de septiembre de 2020.

* Doctor en ciencias sociales. Universidad Autónoma del Estado de México.

Correo electrónico: renebufi@yahoo.com.mx

Pedroza Flores, René. «Estudios del futuro para comprender la posibilidad de la universidad ubicua.» Interdisciplina 9, $\mathrm{n}^{\circ}$ 25 (septiembre-diciembre 2021): 209-231.

DOI: https://doi.org/10.22201/ceiich.24485705e.2021.25.79974 
se presente en todos lados al mismo tiempo para una multiplicidad de sujetos conectados digitalmente de forma simultánea en su singularidad. La universidad ubicua, se concluye, será un cerebro de inteligencia aumentada (inteligencia social más inteligencia artificial).

Palabras clave | prospectiva, futurología, deconstrucción, post-humanismo, inteligencia artificial.

\section{Introducción}

PENSAR SOBRE EL FUTURO de la vida siempre ha inquietado la mente humana, la adivinación, la clarividencia, la predicción, la anticipación y la prospectiva han sido formas históricas de visualizar el destino, lo que está más allá del presente para predecir, prevenir, prepararse o anticiparse. Distintos saberes y conocimientos se han desarrollado para conocer el futuro, unos mágicos y metafísicos, y otros, científicos: Paracelso (2001) habla de las falsas artes de la predicción (nigromancia, necromancia, geomencia, hygromancia) y de las artes inciertas (entre las que se ubica la Cábala); Aubier (1992) escribe sobre las artes adivinatorias (astrología, numerología, cartomancia, tarot, quirología, geomancia, cifrología, radiestesia); Asimov (2000), López-Pellisa (2018) y Fernández (2005) escriben sobre ciencia ficción, término que acuñó en 1926 Hugo Gernsback, también se le conoce como la literatura de anticipación, ficción científica, fantaciencia; y Shuttleworth (2009), aborda el tema de la predicción científica, que proviene de la investigación basada en el método científico.

La ciencia de la previsión y predicción ha evolucionado en el siglo XXI, ha pasado de la visión planificadora moderna de mundo posible o esperado a la visión de prospección posmoderna de mundos alternativos, una nueva disciplina científica se consolida, los estudios del futuro. La importancia de conocer el futuro ha crecido en los últimos tiempos ante el desarrollo científico y tecnológico, y frente a los riesgos planetarios ahora se suman los estudios técnicos de pronósticos, los estudios del futuro con una visión del mundo de mayor alcance, se habla de más de 10 o hasta de 100 años. De la ciencia predictiva, que va del presente al futuro con la finalidad de predecir y comparar, se plantea ahora problematizar las unidades de análisis con la finalidad de construir socialmente futuros alternativos. Por ejemplo, la predicción con métodos y técnicas de la econometría o del análisis de regresión lineal o múltiple analiza la tendencia del crecimiento de la población y su relación con los recursos naturales para determinar esenarios probables o posibles; a diferencia, los estudios del futuro problematizan y deconstruyen las categorías de población y de recursos naturales para construir socialmente escenarios alternativos y distintos:

[...] La planificación pretende controlar y cerrar el futuro, mientras que los estudios del futuro lo que pretenden es abrirlo, avanzando desde 'el' futuro único hasta los futuros alternativos. (Inayatullah 2012a, 40) 
Los gobiernos y las corporaciones en el mundo demandan nuevas formas de ver el futuro porque la constante es el cambio en una realidad global multipolar, la unipolaridad de la geoeconomía dominada por Estados Unidos de Norteamérica se ha fragmentado con los avances científicos, tecnológicos, económicos y militares logrados por China, Rusia, Corea del Sur e India, principalmente, y por el avance en las economías emergentes de distinos países pertenecientes a los diversos continentes. En la aceleración del cambio el factor del desarrollo industrial está siendo clave, las revoluciones industriales tienen menor duración de existencia, las tres primeras tardaron en cambiar más de cien años, las dos últimas, tienen un ritmo de duración sorpredente, entre la cuarta y quinta revolución industrial se estiman dos décadas, las caracterizan la digitalización y la economía circular, respectivamente. Incluso se vislumbra en un futuro mediato la sexta revolución industrial, el tiempo de la singularidad con la fusión hombre-máquina.

En este contexto, la universidad como generadora de conocimiento científico y tecnológico tiene que enfrentar los retos del cambio, prepararse para un futuro que está en construcción permanente. El futuro de la universidad es previsible, si no cambia conforme avanzan las exigencias sociales, su extinción como institución milenaria de formación profesional será una realidad, y si se transforma la universidad del futuro será muy distinta a la universidad actual de paredes, ladrillos y cables. La universidad está frente a la disyuntiva de perecer o reinventarse. Hoy, después de cien años de existencia, la universidad por primera vez en su historia se encuentra en los umbrales de su nueva era: ¿cómo será la universidad del futuro?, ¿cómo nos pensamos la generación y transmisión del conocimiento en el futuro desde la epistemología de la deconstrucción?, ¿qué tipo de aprendizajes y enseñanzas promoverá la universidad? Son algunas interrogantes que inquietan en nuestro tiempo circunstancial.

Los estudios del futuro de la universidad adquieren sentido para comprender y explicar la nueva era de su existencia. Los estudios de prospectiva de la universidad tienen que ser completados con estudios del futuro con los propósitos de: indagar, imaginar, especular en torno de las formas alternativas del mundo de la universidad. Ahora no es suficiente la idea de reformar la universidad, no se trata de reparar o adaptarse únicamente, la finalidad es construir futuros alternativos de la universidad, deconstruir el concepto milenario de universidad para construirla en su nueva era.

\section{Los estudios del futuro}

La futurología ha estado presente a lo largo de la historia, pensar en el futuro siempre ha sido parte de las inquietudes humanas, el futuro como presagio, adivinación, impredicible, ineludible, incierto, predeterminado. No es posible con- 
cebir la vida humana sin pensar en el futuro. Las fuentes del pensamiento del futuro han sido diversas: metafísicas (inferencias), mágicas (adivinación), filosóficas (devenir), religiosas (creación) y científicas (acción). Hasta el presente se mantienen estas formas de imaginar, visualizar, ensoñar, predecir, planear el futuro. Me interesa el abordaje científico de la futurología, como cuerpo de conocimiento epistémico, teórico y metodológico. En esta dirección se ubican los estudios del futuro como una disciplina científica que está en construcción, sus antecedentes sistemáticos y formales se remontan al siglo pasado.

H.G. Wells, reconocido como pionero de la ciencia ficción, aportó una de las primeras referencias contemporáneas en torno de los estudios del futuro: En 1901, escribió el libro Anticipations of the reaction of mechanical and scientific progress upon human life and thought, donde menciona el concepto forecast (previsión), como una forma de pensar el futuro del siglo XX, argumentó que con base en la especulación de las tendencias existentes en el tiempo presente se puede prevenir el futuro; en 1902, escribió un discurso presentado en The Royal Institution: The discovery of the future, donde mencionó la existencia de dos maneras distintas de pensar el futuro: una que asume una visión del futuro con referencia al pasado, y otra que toma conciencia del futuro como porvenir, como hábito constructivo de prevenir a partir de interpretar las cosas del presente. En 1903, escribió el libro Mankind in the making, continuó con el argumento de prever el futuro, en este texto plantea una teoría general del desarrollo social, la conducta social y política como un esquema evolutivo universal de la sociedad. H. G. Wells, consideró esta trilogía en su conjunto como un ensayo en torno del estudio del futuro, colocó el futuro como objetivo de la reflexión humana.

Ossip Kurt Flechtheim, en los años cuarenta del siglo pasado, propuso un nuevo campo de conocimiento que denominó futurología. En su extensa obra asume una postura crítica de mirar el futuro como teodicea u odisea, analiza las posturas del futuro en Hegel, Marx, Toynbee, Weber, entre otros. Ubica la futurología como una división de la sociología porque se encarga del futuro de la civilización que incluye pensar sobre los problemas del destino de la humanidad, al respecto escribió:

Dado que la futurología abarca el destino del hombre, el futuro de su sociedad, y el mañana de su cultura, debe tratar no solo con la prospectiva de la evolución biológica y psicológica, sino también, con toda la gama de sus actividades culturales [...]. La futurología intenta responder de la manera más objetiva posible el problema del destino de nuestra civilización en los próximos siglos [...]. La función de la futurología se hará completamente evidente solo a medida que crezca, ya que sus hallazgos, más que los de otras ciencias sociales, contienen elementos de considerable incertidumbre. (Flechtheim 1966, 76-78) 
A diferencia de otros autores (Solano 2001; Vergara 2010) considero que el aporte de Flechtheim no se reduce el haber bautizado un campo de conocimiento con el nombre de futurología, aportó una visión científica del futuro colocándola dentro de la sociología determinando con claridad su objeto de investigación: los problemas del destino de la humanidad considerando la incertidumbre en toda la gama de actividades culturales, superando así la visión lineal de felicidad y progreso anclada en la teodicea y en la odisea.

En la misma década de los años cuarenta, Herman Kahn, estratega militar de la guerra nuclear — quien trabajó en la Corporation RAND a lado de Edwar Teller, John von Neuman, Hans Bethe y Albert Wohlstetter en el desarrollo de la bomba de hidrógeno- realizó aportes en la planeación militar y aplicó nuevos métodos y técnicas: método de Monte Carlo (aplicación de ecuaciones integrales a través del muestreo aleatorio con el recurso de algoritmos computacionales), teoría de juegos (el estudio de cualquier situación de conflicto donde hay más de dos actores con objetivos distintos), juego de guerra (determinación de las relaciones, reglas, reacciones y pruebas de maniobra entre enemigos), creación de escenarios (secuencias hipotéticas posibles de conducir a situaciones previstas), investigación de operaciones y análisis de sistemas (diseño de la ofensiva y la defensiva en las guerras bilaterales con el uso de la probabilidad). El aporte de Herman Kahn consistió en dar sustento metodológico y técnico al estudio del futuro con una visión estratégica en la creación de escenarios.

Durante las siguientes tres décadas, de los años cincuenta a los años setenta, las aportaciones se multiplicaron, son los años dorados de la futurología, una pléyade de futurólogos de distintos países entraron en escena: Gaston Berger quien propuso el término prospectiva (Francia), Michel Godet (Francia), Bertrand de Jouvenel (Francia), Pierre Massé (Francia), Pietro Ferraro (Italia), Roberto Vacca (Italia), Aurelio Pecci (Italia), Yoneji Masuda (Japón), Hidetoshi Kato (Japón), Willis Harman (norteaméricano), Joseph Hodara (México), Tomas Miklos (México), entre otros. Se fundaron organizaciones e institutos dedicados a la investigación del futuro: Centro Internacional de Prospectiva, Asociación Internacional Futuribles, Instituto Hudson, The World Future Society, Club de Roma, The World Futures Studies Federation (Medina 2006a y 2006b). La futurología científica tiene un pasado reciente:

Los estudios del futuro son un producto moderno por excelencia; un fenómeno concomitante con la diferenciación de las esferas institucionales, la creencia en la idea de progreso, la fe en la ciencia y el poder tecnológico, la secularización, el nuevo papel del individuo, la actitud positiva respecto al futuro, la aceptación entusiasta del cambio y la transformación de las concepciones de autoridad. Por ende, esta concepción moderna dista mucho de las visiones pre-modernas, fundamentadas en actitu- 
des místicas, mágicas, fanáticas, rígidas o conservadoras, donde prima el temor, el azar y la fatalidad. (Medina 1996, 5)

En la concepción moderna de los estudios del futuro identifico tres puntos relevantes en su etapa inicial: primero, fue una época donde quedaron asentados los fundamentos, métodos y técnicas del estudio del futuro, las visiones transcurrieron entre un futuro pesimista y un futuro optimista: por un lado, la corriente neomalthusiana del crecimiento demográfico exponencial frente al crecimiento aritmético de los recursos, postura sostenida por el Club de Roma con su diagnóstico pesimista en su obra crucial: Los límites del crecimiento, y, por el otro lado, la corriente de la innovación basada en los ciclos de Kondratiev, que argumenta la existencia de oleadas de expansión y recesión de una duración de aproximadamente 25 años. Segundo, el estudio del futuro se consolidó científicamente, marcaron un hito en la historia conceptos como anticipación, prevención, prospectiva, estrategia, acción, pronóstico. Tercero, el florecimiento de abordajes metodológicos cuantitativos y cualitativos provenientes de las ciencias sociales, las humanidades y de las matemáticas: Delphi, análisis de contenido, análisis de series de tiempo, modelación, simulación, análisis de impacto cruzados, analogías históricas, juicios de expertos, mapas conceptuales del futuro.

En las décadas posteriores, de los años ochenta hasta el presente, se han fortalecido los estudios del futuro pese a críticas que carecen de sustento científico al reducir la aportación de estos estudios a intuiciones sin fundamento. Lo cierto es que desde sus inicios los estudios del futuro han tenido un fundamento sociológico, matemático y humanista que con el tiempo se ha ido diversificando interviniendo otras disciplinas científicas que le han proporcionado teoría, métodos y técnicas. En las últimas cinco décadas, se ha ido perfilando un cambio en la concepción de los estudios del futuro, del énfasis de predecir se ha pasado ahora a centrar la atención en la exploración de futuros alternativos y a configurar futuros deseados (Forward Thinking Platform 2016). Se apertura una mirada crítica del estudio del futuro para superar el exceso de la linealidad de la idea del progreso y de la especulación catastrófica, se trata ahora de deconstruir los metarrelatos del único futuro creando genealogías discursivas, que cuestionen el relato dominante y exploren los otros relatos que quedaron subsumidos en la historia y en el presente. Uno de los actuales teóricos que sustenta la postura crítica de los estudios del futuro es Inayatullah, quien expresa esta tendencia reciente en las palabras siguientes:

Los estudios críticos del futuro están inspirados en el postestructuralismo y tienen por tarea convertir lo universal en particular, demostrar que han sucedido por motivos de fragilidad política, simplemente la victoria de un discurso sobre otro, no es un 
universal platónico. Para lograrlo, se necesitan genealogías discursivas que intenten demostrar las discontinuidades en la historia de cualquier idea, formación social o valor. Mediante la genealogía y la deconstrucción, el futuro que antaño parecía inmutable se muestra ahora como uno de tantos. Como tal se puede sustituir por otros discursos. A continuación, la deconstrucción se convierte en un método para sacar de la maleta un texto (definido ampliamente) y mostrar los discursos que habitan en él. La deconstrucción va más allá del relativismo, al preguntarse cuál es el precio de un discurso concreto: ¿Qué futuro hemos establecido? ¿Qué futuro hemos silenciado? (Inayatullah 2012, 46)

Como señala Inayatullah al deconstruirse la noción de futuro se abre un abanico de posibilidades de concebir, estudiar, desear, imaginar y construir el futuro socialmente. El conocimiento del futuro es esencial, quizá ahora más que antaño, porque la sociedad cambia con mayor dinamismo que en el pasado; por ejemplo, las primeras revoluciones industriales eran de larga duración, alrededor de 100 años, mientras que entre las dos últimas su tiempo de duración se acortó significativamente a menos de la mitad que el de las primeras.

En este escrito, asumo la visión actual de los estudios del futuro para comprender la dinámica de la universidad. La universidad en sus diez siglos de existencia no se había enfrentado a una situación adversa como la actual, está en riesgo su existencia en el futuro si no se transforma. Los actores universitarios gestores, docentes e investigadores posiblemente sean una especie en extinción frente a las inercias y los cambios lentos que ahora se emprenden. No se requieren más reformas universitarias de corto y mediano plazo basadas en indicadores de calidad para satisfacer los egos académicos con los rankings. La transformación como condición de existencia en el futuro exige pensar la universidad desde los estudios del futuro para que se pueda hablar de la universidad del futuro y no de la duda ni de la incertidumbre del futuro de la universidad.

La reflexión en torno de la universidad del futuro a través de los estudios del futuro ofrece la posibilidad de deconstruir el relato dominante de universidad que está en boga y que tiene sus raíces en la racionalidad económica y planificadora desde mediados del siglo pasado. La planeación para la eficiencia externa e interna vinculada con el postulado del desarrollo del país, el discurso dominante fue el hacer uso eficiente de los recursos materiales, financieros y humanos como previsión. La idea de futuro estaba anclada en la prevención sistemática y permanente como medio de garantizar la educación universitaria. Por ejemplo, en un artículo de los años setenta se escribía de la forma siguiente:

La planeación de la enseñanza superior constituye, como proceso, una previsión sistemática y permanente del futuro desarrollo del sistema, en función de la determina- 
ción de los requerimientos de recursos humanos de alta calificación del país. Su objetivo central es el de satisfacer esos requerimientos, logrando la mayor eficacia y eficiencia en la utilización de los recursos humanos, materiales, económicos y técnicos que, para tal efecto, la sociedad canalice hacia el sistema. (Domínguez 1972, 2)

El futuro se concebía como manejable, en una visión lineal sin inestabilidad, ni incertidumbre y sin complejidad, era un futuro esperado que se podía prever, era algo esperado y controlado. Está visión veinte años después continuaba en el tintero de los estudios sobre la universidad, en un artículo del año noventa se escribía:

Para concebir la universidad del futuro, tenemos necesariamente que considerar las características imaginables de la sociedad de los años venideros. Para plantear estos caracteres, el estudio o análisis debe partir del conocimiento de la realidad presente y de sus proyecciones o tendencias; luego entonces, lo que procede es un diagnóstico de la universidad actual para explicar o discutir los atributos deseables de la universidad del mañana, de acuerdo o en armonía con la sociedad de entonces [...]. (Rivera 1990:1-4)

El futuro continuaba siendo parte de la planeación y de la prospectiva, se concebía de forma lineal, a partir del diagnóstico se visualizaba la prospectiva. Como se señala en la cita, con la planeación se facilitaba alcanzar el futuro. La planeación durante largo tiempo ha estado presente como forma de controlar el futuro de la universidad, una planeación que prevé con medición y control la universidad del futuro. A la idea de planeación se sumaron los principios de evaluación y de la calidad, los horizontes del futuro de la universidad quedaron anclados a la idea de una universidad de calidad que recibió distintos nombres: universidad emprendedora, universidad innovadora, universidad flexible, universidad competitiva, universidad industrial. Así, las reformas universitarias realizadas a lo largo de las últimas siete décadas tienen el sello del estudio del futuro como prevención.

La concepción de futuro de la universidad toma un giro al conceptualizarse de forma distinta en los últimos años a partir de los cambios tecnológicos y de los desafíos mundiales de la sociedad actual (Chung 2012; Gómez 2019; Inayatullah 2000). El futuro se concibe ahora con incertidumbre, donde es posible construir futuros alternativos a partir de deconstruir el concepto monolítico de universidad. Estamos ante un parteaguas de la universidad porque su transformación es necesaria para que continúe su existencia en otra forma distinta a la actual, la tendencia es marchar hacia la universidad disruptiva. La universidad está presionada por distintas fuerzas sociales que le obligan a revitalizarse como condición de existir en el futuro. 
El Departamento de Educación de Norteamérica a través de la Comisión sobre el Futuro de la Educación Superior publicó un informe donde resalta la necesidad de transformar la universidad ante la dinámica de los cambios para poder contar con una institución que pueda ser líder en el futuro:

El futuro de los colegios y universidades de nuestro país está amenazado por las presiones competitivas, desarrollos tecnológicos, restricciones financieras y limitaciones estructurales que demandan reformas. Nuestro informe recomienda acciones estratégicas diseñadas para mejorar la educación haciéndola más accesible, equitativa y responsable, manteniendo una calidad de clase mundial. Nuestros colegios y universidades deben transformarse más rápido para responder a las circunstancias que cambian rápidamente [...] para enfrentarse decididamente con las poderosas fuerzas de cambio existentes ahora. (U.S. Department Education 2006, 27)

El caso de la situación de la universidad norteaméricana ilustra la situación de la universidad en el mundo, las fuerzas sociales presionan y demandan transformar la institución milenaria en una universidad activa y dinámica para enfrentar el futuro. La universidad tiene ahora la responsabilidad de realizar profundos cambios paradigmáticos de su estructura y función interna y en su forma de relacionarse externamente con la sociedad. Desde los estudios del futuro con los aportes de Inayatullah se plantea que la universidad pasa por un proceso de transformación donde el futuro se lee de forma distinta a como se leía en el pasado con la prospectiva, ahora se aperturan nuevas lecturas con narrativas que provienen de distintas disciplinas sociales y de la ciencia ficción.

\section{Las presiones sobre la universidad}

Sobre la universidad pesan dos presiones intimimante vinculadas entre sí y que impactan en las prácticas y en el modelo académico: por un lado, los desafíos mundiales y el cambio científico y tecnológico. En los desafíos mundiales se ubican problemas no resueltos en la historia contemporánea de la sociedad: la pobreza, la desigualdad, la inequidad, la violencia en todas sus formas, y los problemas emergentes: el agotamiento de los recursos naturales, el terrorismo ecológico, el calentamiento global, el problema de la basura, la economía capitalista de consumo irracional. Por el otro lado, el cambio científico y tecnológico tiene un crecimiento rápido e inédito que impacta en la forma de vida social en todos los aspectos: económico, laboral, social, cultural, etcétera. Estas dos presiones sociales acompañan a la universidad en su transformación. En un documento reciente se señalan los cambios que están dando rostro al futuro: 
En los próximos 35 años se espera que se sumen al planeta otros 2,300 millones de personas. En 2050, se necesitarán nuevos sistemas para la alimentación, el agua, la energía, la educación, la salud, la economía y la gobernanza mundial para prevenir, de manera masiva y compleja, desastres humanos y ambientales. La investigación de futuros del Millennium Project muestra que la mayoría de estos problemas se pueden prevenir y que es posible un futuro mucho mejor que el de hoy [...]. Las interacciones entre las futuras inteligencias artificiales, un sinnúmero de nuevas formas de vida de la biología sintética, la proliferación de los ensamblajes nanomoleculares y la robótica podrían producir un futuro apenas reconocible por la ciencia ficción de hoy. (Glenn $2015,1)$

Estos cambios impactan en el presente y futuro de la universidad, porque como institución que conserva, regenera y crea conocimiento debe ser partícipe de las soluciones que se demandan por parte de la sociedad. Para efectos de este escrito me interesa en particular ahondar en el desafío de ciencia y tecnología por tres razones: impacta a toda la sociedad, transforma a la universidad y cambia los estilos de ser, pensar y relacionarse de todos. La sociedad con el desarrollo científico y tecnológico tiende al transhumanismo (convivencia regulada por humanos con otras especies no humanas) o al posthumanismo (convivencia regulada por especies no humanas sobre los humanos) según sea el enfoque que se asuma en el futuro de la sociedad, la universidad tiende a un cambio paradigmático que cuestiona su sistema actual, y en los procesos de enseñanza y aprendizaje no solo formales transforman las mentalidades, comportamientos y acciones de todas las personas.

Jordin Pigem (2018) escribe que hay que observar la realidad con sinceridad, lo cual representa la oportunidad de aceptar que estamos viviendo el final de una era, el final del mundo moderno. Ante la nueva era, expresa este autor retomando a Panikka, se requiere una profunda transformación de la consciencia humana nombrada como metanoia (meta: más allá, nous: mente). Pigem concibe la metanoia como una forma distinta de ver el mundo, una realidad entrelazada que supera las controversias y los autoengaños humanos que han conducido al deterioro en las condiciones y formas de vida material y subjetiva. Nunca antes habíamos estado frente a un cambio de repercusiones inciertas para el ser humano con el desarrollo científico y tecnológico que abre un abánico de posibilidades de futuro entre las tecnoutopías y las tecnofobias.

En este contexto de transformación de la sociedad en su conjunto, la idea que presento - con base en lo expuesto hasta el momento- es que la universidad está llegando al final de una era, la era de la universidad de ladrillos. Los umbrales de la nueva era de la universidad se caracterizan por el dinamismo que tienen los desarrollos científicos y tecnológicos en todos los campos del conocimiento. 
La universidad ha empezado a dejar de ser lo que era antes: la catedral del saber; la certidumbre de su progreso en la formación de profesiones modernas y de su trabajo fragmentado de investigación están siendo cosas del pasado junto con su estructura jerárquica y vertical de gobernanza que limitaban la libertad de conocimiento como creación y como práctica intra y extramuros de la universidad.

En este cambio de era, la ciencia y tecnología tienen un papel revitalizador, no existe lugar de la universidad que esté libre de big science, tecnociencia y tecnologías del aprendizaje. Presenciamos una simbiosis entre especies naturales y no naturales, o como la denomina López, una fusión humanidad y tecnología que conforma el binomio humanización-cyborg: "la tecnología se fusiona con el cuerpo, con la mente, y funciona como herramienta" (López 2017, 11). Existe un proceso de fusión entre la inteligencia humana y la inteligencia artificial, humanos creando especies no humanas que están generando un escenario de sociedad que solo había sido vislumbrado por la ciencia ficción.

Los avances científicos en todos los campos del conocimiento presionan a la universidad para transformarse ante los umbrales de la nueva era de la singularidad. Hoy es mucho más rápido el cambio científico y el desarrollo tecnológico que en los siglos anteriores. La biología durante las últimas dos décadas está en un proceso inédito de avance, se están creando organismos sintéticos inaugurando la creación de una nueva naturaleza que se desliza entre el genoma humano y el genoma tecnológico; la física del siglo XXI se caracteriza por sus avances que apuntan a distintas direcciones en el mundo microscópico con la física de partículas y en el mundo macroscópico con la astrofísica, pasando por la física computacional, física nuclear, física de bajas temperaturas, física de plasma, física del estado sólido, entre otras físicas y temas teóricos y experimentales, que están cambiando nuestra realidad hacia la nanotecnología incorporada en el organismo; la química actualmente avanza con sus aportaciones hacia las máquinas moleculares, la nanociencia, a la conversión de la energía y a la creación de nuevos materiales; las ciencias cognitivas han aportado un cuerpo teórico interdisciplinario para explicar el funcionamiento de los estados mentales, uno de sus presupuestos es la mente extendida, la dualidad de mente-cuerpo, al separar el cerebro de la mente se abre la posibilidad de explicar la mente como separada del cuerpo, colocando la idea de que el ser humano es un cyborg y aportando elementos fundamentales en la robótica.

El cuerpo y las funciones humanas son ahora impensable sin la incorporación y convivencia con dispositivos y artefactos tecnológicos que derivan de los logros científicos. El hombre mejorado que en la década de los sesenta, Manfred E. Clynes y Nathan S. Kline bautizaron con el nombre de cyborg, y que Haraway lo definiera como: "Un organismo cibernético, un híbrido de máquina y organismo, una criatura de realidad social y también de ficción” (1984, 1). Esta criatura 
ha evolucionado en el siglo XXI con dos mentes, la orgánica y la máquina: "Una de sus mentes, la orgánica, está constantemente tratando de aprender, de crear cosas nuevas, de experimentar y hasta de equivocarse. La otra mente, la máquina, trata de ser efectiva, rápida, exacta, perfecta. Mientras una se encuentra buscando la otra se encuentra realizando. Mientras una se encuentra viviendo la otra se encuentra redefiniendo lo que es la vida en el siglo XXI" (Monje y Ortegón 2016, 7). Con el desarrollo de la nanotecnología, la nonomedicina, la biomimética, la biónica, la inteligencia artificial, se fortalece el mundo cyborg. Yehya describe este mundo de la forma siguiente:

Hoy se habla cada vez con más seriedad de un futuro en el que se nos implantarán chips para mejorar nuestra memoria, así como dispositivos de comunicación (internet intercraneal y telefonía microcelular) y aparatos para monitorear nuestra salud o simplemente para funcionar como interfaces de las tecnologías que nos rodean. Un circuito integrado debajo de nuestra piel podría emitir una señal o ser leído con un escáner para servirnos de pasaporte al atravesar fronteras, de llave para acceder a nuestra computadora o para hacer que se enciendan y apaguen las luces de nuestra casa u oficina al movernos de una habitación a otra. Otra tecnología que espera rendir frutos en las próximas décadas es la nanotecnología [...], que consiste en manipular materiales a nivel molecular para crear nanomáquinas y con ellas fabricar cualquier cosa, lo mismo un tomate que una nave espacial. (Yehya 2013, 1)

Lo que escribe Yehya ya es una realidad. Neil Harbisson es un ejemplo, desde el año 2004, está reconocido oficialmente en el mundo como el primer cyborg (unión entre cibernética y organismo); como él mismo lo expresa, es tecnología; lleva colocado un dispositivo que le permite superar su cromatismo visual (ver en escala de gris) que le limitaba ver los colores, lleva una antena implantada en la cabeza que le permite escuchar la frecuencia de los colores visibles e incluso de los colores infrarojos hasta los colores ultravioletas que el ojo humano no es capaz de percibir, además de realizar otras funciones como recibir llamadas telefónicas y captar señales de satélite. Neil Harbisson expresa que la tecnología ha dejado de ser un dispositivo externo en el humano, la tecnología se ha incorporado no solo en el cuerpo, también está colocada en la mente, la tecnología ha modificado nuestra psicología, nuestra convivencia social; para él, estamos en una transición de humanos con tecnología a cyborg biológicos que amplían sus percepciones, capacidades y habilidades.

Se espera que la evolución de los cyborg en el siglo XXI arribe a la inmortalidad del ser humano al incorporar el cerebro humano en el cuerpo de un robot, este es el objetivo del Proyecto Avatar hacia el año 2045, que promueve el ruso Dimitry Itskov desde el año 2012. Es un proyecto dividido en cuatro fases: Avatar 
A, construir un robot controlado por la mente humana (2015-2020); Avatar B, implantar el cerebro de una persona a un avatar robótico (2020-2025); Avatar C, trasladar el contenido de un cerebro humano a un soporte artificial (2030-2045); Avatar D, crear una vida virtual que significa la existencia holográfica de una persona, la persona viviendo íntegramente al interior de un sistema computarizado. Existen dudas de poder lograr esto en el plazo planteado por Dimitry, independientemente de estas críticas, el aporte de este ruso es plantear la tendencia del desarrollo de los cyborgs en un futuro no muy lejano.

Junto con la evolución de la especie humana están en desarrollo otras especie no humanas, como lo robots. En la mente y en las acciones humanas los robots no son algo nuevo, han estado presentes desde la antigüedad, existen muchos ejemplos: el actor de teatro construido en el año 85 por Herón de Alejandría, el hombre de Hierro de Alberto Magno (1204-1282), el Gallo de Estrasburgo (1352), el León mecánico de Leonardo da Vinci (1499), el trío de autómatas (el dibujante, la pianista y el escritor) de Jaquet-Droz (1768-1774), la muñeca dibujante de Henry Maillardet (1805); así sucesivamente hasta llegar a desarrollos más complejos como: Don Cuco el Guapo, el primer robot-humanoide pianista diseñado y construido en 1992 por un grupo de científicos mexicanos de la Benemérita Universidad Autónoma de Puebla: Alejandro Pedroza, Javier Méndez, Ignacio Becerra, Héctor Simon, Jorge Sánchez, Eduardo Oceja y Daniel It; y el robot pianista Rohmus, diseñado en 2014 por Miguel Adad Martínez del Instituto Politécnico Nacional; también Sophia, la robot más humana que cuenta con ciudadanía del mundo.

La robótica ha pasado por cuatro etapas a lo largo de su historia: sistemas mecánicos, sistemas de sensores, sistemas programados, sistemas inteligentes y sistemas de subsunción. En la robótica participan distintas especialidades científicas de forma interdisciplinaria: microelectrónica, cibernética, nuevos materiales, electromecánica. En este siglo XXI, la robótica ha adquirido relevancia, según datos de la International Federation of Robotics (IFR), se calcula la población de robots en 2019 en 2.6 millones de robots y se espera que para 2020 se instalen más de 1.7 millones de robots. El crecimiento mayor de robots se registra en Asia y después en USA y en Europa. Los robots están presentes en distintas áreas de la sociedad, donde mayor es su presencia es en la industria, con datos del 2017 de la IFR por cada 10,000 empleos hay 710 robots en Corea del Sur, 658 en Singapur, 322 en Alemania, 308 en Japón.

Los robots están presentes en la educación (Aoun 2017; Ribeiro 2020; Varela 2020; Manera 2020), como apoyo del ecosistema de aprendizaje, asistentes de profesores y como profesores, la empresa SoftBank Robotics creó los robots Pepper y Nao, que intervienen en el aprendizaje en la primaria y en la universidad. Pepper es un robot humanoide con inteligencia emocional que le permite estudiar con los estudiantes, se ha utilizado en distintas clases: en la clase de lengua del 
Instituto Shoshi de Waseda (Japón); en la educación superior en las clases de negocios internacionales en la ESADE Business School, de Barcelona; en la London Design \& Engineering, en Francia apoya el aprendizaje de la gramática. No solo los profesores se están robotizando, también los estudiantes, la empresa Double Robotics aporta robots para la clase que dirije Bill McCaw en la Universidad de Montana (Estados Unidos de América), los estudiantes se ponen en contacto entre sí a través de robots que ruedan con una pantalla instalada; un caso similar es el aprendizaje híbrido en Michigan State University, que utiliza la robotic telepresence. Estos son solamente algunos ejemplos de muchos más de la presencia de los robots en la educación que vienen a transformar la dinámica del aprendizaje:

La predicción de que en un futuro próximo los robots inteligentes sustituirán a las personas en todos los puestos de trabajo hasta ahora no se había referido a aquellas profesiones en las que se da una interacción personal, pero eso está empezando a cambiar. El sector educativo ya está empezando a sentir los efectos de la explotación de datos para imitar el comportamiento de los estudiantes y desarrollar sistemas de tutorización inteligentes. (Gómez 2019, 1)

La primera era de la universidad de humanos para humanos está en proceso de transformación hacia una nueva era de relaciones entre la especie humana y las especies no humanas. Ahora estamos en un proceso de transición que se caracteriza porque la universidad tiene que responder a los desafíos mundiales de tipo social y al desafío de la ciencia y tecnología. Por el lado social, la universidad tiene que ser partícipe de la justicia social a través de la justicia académica, para impulsar el sentido humano con respecto a la naturaleza y a todos los seres vivos; y por el lado científico, la universidad tiene que impulsar el desarrollo de la ciencia y la tecnología y a su vez incorporar procesos académicos inéditos no del todo imaginables en el presente. Socialmente las relaciones académicas en la universidad se transforman desde la célula básica que son las tareas de aprendizaje hasta la estructura que deja de ser únicamente física y trasciende a lo digital. El estudio del futuro de la universidad se hace ahora con una visión crítica y no únicamente lineal, lo que posibilita pensar la universidad como un entramado tejido entre lo tradicional y lo emergente.

\section{El futuro en y para la universidad}

El futuro es un desafío que pesa sobre la universidad de dos maneras distintas: la primera, ¿la universidad existirá en el futuro o su fin está próximo?; la segunda, de mantenerse en el futuro la universidad ¿cómo será la universidad del futuro? Si se consideran los estudios del futuro en su versión actual que tienden a 
deconstruir la certeza conceptual y a construir futuros alternativos, la universidad tradicional-moderna (la que tiene 10 siglos de existencia) está en proceso de extinción, su arquitectura académica es un dinosaurio que se mueve lentamente ante la corrupción, inercia, resistencia e ignorancia de todo tipo, ante esto desprendo un par de tesis: la primera tesis, universidad que no se transforme está condenada a su extinción, no todas las universidades en el mundo sobrevivirán, no podrán evolucionar a una nueva era, todo dependerá de su disposición y capacidad para transformarse, Clayton Christensen preveé que la mitad de universidades dejarán de existir en 10 años; la segunda tesis, la nueva era de la universidad estará marcada por la convivencia académica entre humanos, cyborg y robots con procesos académicos inéditos.

Hoy en día, no hay universidad en el mundo que no esté preocupada y ocupada en el tema de su futuro. Ahora que la universidad ha dejado de ser, ya desde hace tiempo, el monopolio del saber, debe competir con nuevos actores que aparecen en la escena del mercado del capitalismo académico. Las universidades tienen que competir con contendientes mejor preparados económicamente y que están dispuestos a llevar a cabo un proceso de disrupción universitaria. Las universidades, las públicas principalmente, que dependen del presupuesto estatal se encuentran muchas de las veces en condiciones desfavorables en materia de competitividad porque se les dificulta realizar procesos de innovación trascedentes. Por ejemplo, mientras las universidades tradicionales continúan en la dinámica de lo presencial otros actores están apostando a otras modalidades con los recursos de las tecnologías aplicadas a la educación.

Eyring y Christensen (2011) aseveran que está cambiando el ADN de la universidad, lo expresan desde su posición de estudiosos del mercado, al integrar la variable productividad-competitividad, donde la universidad como organismo que evoluciona debe considerar la mutación de sus genes para estar no solo mejor adaptada sino, además, debe crecer y mejorar la escala de su alcance. Inayatullah (2000), desde otro ángulo, señala que la universidad está en transformación impulsada en especial por cuatro factores: globalización, multiculturalismo, virtualización y politización; este especialista en estudios del futuro, visualiza la tendencia hacia universidades corporativas megaglobales coexistiendo con universidades pequeñas diversificadas atendiendo demandas locales derivadas del muticulturalismo (universidades de mujeres, universidad espiritual, universidad indígena); llama la atención por parte de este autor, que habrá un debilitamiento de la presencia estatal en la universidad y que habrá un debilitamiento de la libertad política ante la creciente virtualidad. Don Tapscott (2017), argumenta que la revolución digital desafiará a la universidad en su forma tradicional; al igual, Peter Druker piensa que las universidades en 30 años serán reliquias que no lograrán ponerse al día porque tienen un atraso de 100 años, las tecnologías que cambiarán radical- 
mente la universidad son las llamadas blockchain (segunda generación de internet) que conducirán a la universidad del siglo XXI a ser una red y un ecosistema, no una torre como ahora aún lo es.

Este conjunto de autores, desde ángulos diferentes, coinciden en que está en marcha un cambio radical en la universidad: una universidad con un ecosistema tecnológico de dimensiones globales y alcances locales compitiendo en el mercado de la educación superior haciendo uso de la segunda generación de internet orientada a la innovación disruptiva. Sin duda, esta tendencia está marcada por el énfasis económico y por el debilitamiento de la universidad pública que estará limitada para competir con las megauniversidades comerciales que ofrecerán cada vez más una diversidad de títulos y grados, resultado de itinerarios curriculares abiertos. En este marco de tendencias conceptuales: ¿qué cambios están realizando las universidades?

Rusia se propone impulsar la universidad 3.0 cuyo eje es la innovación en la generación de propiedad intelectual comercializada al ser puente entre la ciencia y los negocios; desde el 2005, se ha generado una corriente de pensamiento que observa la universidad como fuente potencial de innovación y pretende una reforma similar a la universidad norteamericana retomando la experiencia de Silicon Valley (Laptev 2016). En la India y Pakistán, la reforma universitaria da prioridad al aprendizaje a distancia y al modelo de acreditación internacional, para estos países del sur de Asia el mercado de educación superior virtual es una ventana de oportunidad, en el año 2017 representó un ingreso de US \$42,700 mil millones y en el 2018 fue de US $\$ 50$ mil millones; otro dato importante es que la tasa de crecimiento de la demanda de posgrados en línea fue del $17.3 \%$ más alta que en cualquier otro lugar del planeta, en la actualidad cuentan con 3 millones de estudiantes cursando estudios superiores en línea a través de plataformas como edX, Coursera, Udacity, FutureLearn. Estos cambios se describen como una convergencia entre lo tradicional y lo digital:

Muchas universidades también están comenzando a ofrecer modelos de aprendizaje combinados más colaborativos, mediante los cuales a los estudiantes se les enseña usando una combinación de aprendizaje en línea y matrícula presencial en lugar de únicamente a través de conferencias tradicionales. (British Council 2015,3)

La universidad en Malasia transita al modelo de universidad basada en la industria retomando las necesidades de la comunidad, creando el proyecto de universidad 2025, y fortaleciendo su apertura de oferta a la carta porque está en juego un mercado de 2.5 billones de dólares, se espera que la demanda que era de 2,000 millones de estudiantes para el 2025 llegue a 262 mil millones de estudiantes en la demanda pública y en la demanda privada el mercado educativo se esti- 
ma en 400 mil millones de dólares a nivel mundial. Inayatullah se interroga sobre el futuro de la Universidad de Malasia en los términos siguientes:

\begin{abstract}
¿Transitará la Educación Superior en Malasia del modelo de fábrica a un enfoque de "Café" centrado en el estudiante, la universidad "a la carta"? ¿Los profesores seguirán sumidos en formas burocráticas o podrán centrarse en la enseñanza y el aprendizaje? ¿Tendrán éxito las plataformas de aprendizaje combinado? Los actuales impulsos del futuro (las nuevas tecnologías digitales, una sociedad que envejece, los paradigmas cambiantes en el aprendizaje y la mayor globalización) abrumarán la educación superior en Malasia o ¿la Educación Superior de Malasia puede responder a estos impulsos de manera que cumplan con estudiantes, profesores, universidades, industrias y necesidades de la comunidad? (Inayatullah 2012b, 1)
\end{abstract}

La universidad China está en transformación de forma acelerada, le está ganando terreno a Norteamérica y a Europa, en la economía del conocimiento está atendiendo la formación profesional en las áreas más importantes para el desarrollo científico y tecnológico: ciencias, tecnología, ingeniería y matemáticas (CTIM), se calcula que para 2030 China junto con la India formen al 60\% de egresados de CTIM en el mundo, en comparación con el 8\% esperado para Europa y el 4\% de los estadounidenses.

En este escenario el futuro que argumentan los expertos y las acciones que realizan los reformadores universitarios está determinado por los factores siguientes: la competencia por el mercado académico de millones de dólares, la vinculación de la universidad con el desarrollo industrial de las naciones, el posicionamiento científico-tecnológico en la globalidad, la variabilidad de la oferta formativa con modalidades digitalizadas, el desarrollo de innovaciones disruptivas que aperturan nuevas agendas de negocios, solución de las demandas de las comunidades locales y la atención a las formaciones profesionales vinculadas con la economía del conocimiento (ciencia, tecnología, ingeniería y matemáticas). Las universidades en estos escenarios han recibido distintos nombres: universidad emprendedora, universidad innovadora, universidad 4.0, universidad 3.0, universidad de la $4 \mathrm{~T}$, etcétera.

De la forma en cómo se comporten las universidades frente a ese escenario dependerá su futuro. Pulido (2009), con base en entrevistas a expertos y al análisis de los diversos cambios emprendidos por las universidades en el mundo, plantea cuatro escenarios del futuro de la universidad: universidades tradicionales sin cambios, universidad tradicional adaptada, universidad con estrategia nacional/local y universidad con estrategia global. Todo dependerá de cada universidad la estrategia que seguirá con base en su situación, condición y posibilidad de reforma o transformación: 
Ante las múltiples estrategias posibles a adoptar dentro de ese entorno global, se tenderá a una especialización de acuerdo con las ventajas comparativas. Las universidades más potentes podrán captar profesores, alumnos y recursos para investigación de otras zonas geográficas. Algunas incluso podrán abrir campus en el exterior, establecer consorcios u ofrecer franquicias para programas educativos, como ya lo hacen algunas universidades británicas o norteamericanas. (Pulido 2009, 116)

La tipología de la universidad hacia el futuro que plantea Pulido, la ubico dentro del discurso dominante de una universidad puente entre la economía y la innovación tecnológica que contribuya al dominio del poder de una nación o naciones sobre otras dentro de la carrera del conocimiento. Este tipo de posiciones dentro de los estudios del futuro se identifican con la ola que predominó aproximadamente en los años ochenta del siglo pasado, una visión del futuro que supone la línea del progreso sobre la cual todos marchan a ritmos distintos dependiendo de la estrategia diseñada y de sus capacidades y condiciones con las que se cuente. En esta lógica el futuro es un faro que orienta hacia la meta a lograr: la meta de la universidad en el discurso dominante es ganar el mercado mundial del conocimiento y posicionar económicamente a las naciones, por esta razón se habla de universidades que no podrán realizar cambios, otras que se adaptarán a las fuerzas competitivas, unas más que quedarán colocadas en lo regional y otras más que encabezarán el futuro al lograr la meta dominante.

El futuro de la universidad dentro de la línea del progreso tiene formas distintas de plantearse: el futuro lineal al considerar que si se trabaja duro se asegurará la meta del futuro; el futuro cíclico porque en la rueda de la vida unas universidades estarán ahora arriba y mañana quizá abajo; el futuro en espiral: una veces las universidades se sostendrán progresivamente de forma lineal y otras entrarán a la espiral con caídas en el futuro; el futuro bisagra: las universidades como puente entre la tradición y el cambio; y, el futuro como cajita cerrada cuando las universidades se centran en sí mismas como potencialidad de la transformación.

Existe otra forma distinta de ver el futuro, el enfoque de los estudios críticos del futuro, que se propone explorar futuros alternativos y deseados a partir de la deconstrucción del metarrelato dominante y al analizar relatos que han quedado subsumidos en la tendencia dominante. En este sentido, un relato que si bien se ha mencionado en el futuro de la universidad no se ha dimensionado en toda su extensión sus implicaciones para la universidad: es el relato de la digitalización del ser humano que trastoca las certidumbres en las formas de ser, conocer y hacer que son el ADN de la universidad. La existencia de cyborgs, robots y otras especies no humanas que están en proceso de crearse tienen que profundizar en formas inéditas de manejar al conocimiento y a la experiencia científica 
y tecnológica: científicos y tecnólogos creando otras especies híbridas y no humanas que generarán ciencia y tecnología más allá de los límites de las capacidades y de los sentidos humanos. Con base en los estudios del futuro que incluyen la ciencia social y la ciencia ficción se puede construir la narrativa de la realidad aumentada (inteligencia social más inteligencia artificial) que está presente en la universidad. La universidad empieza a estar en todos lados con distintas formas, es una institución ubicua en la era de la singularidad.

Una interpretación alterna al discurso dominante de la universidad de ladrillos está en los estudios del futuro que posibilita una escritura abierta para pensar socialmente la universidad que está por venir (Chung 2012). Una universidad que empieza a estar en todas partes porque se puede acceder a estudios superiores de forma digital y virtual, una universidad ubicua, de la que ahora estamos en sus umbrales, se expandirá en el espacio no físico con terminales en los $c y$ borgs y robots, contando con centrales de exploración del conocimiento. El procesamiento del conocimiento y de la información en las formaciones profesionales dejará de ser el aprendizaje significativo que tanto se menciona ahora en su versión constructivista y de competencias, en su lugar estará la exploración hacia lo desconocido. Las barreraras y las limitaciones naturales del ser humano serán superadas en la universidad ubicua.

La universidad ubicua - la que está presente de forma virtual y digital-en el corto plazo, se caracterizará porque estará en transición entre la vieja y nueva era de la universidad. En el mediano plazo la universidad será un espacio de formación físico y no físico en un rizoma digital entre cyborgs y robots. En el largo plazo, la universidad actual será un museo. La universidad ubicua será el cerebro social del saber y del conocimiento en la sociedad, la frontera entre universitarios y no universitarios dejará de existir, todos podrán acceder al conocimiento, sin dejar de lado, que esto traerá formas inéditas de luchas por el poder, ya que la universidad será el bien más preciado porque representará el dominio de la exploración. La historia del futuro de la universidad ubicua apenas empieza a escribirse, la narración ahora está inconclusa deberá de completarse conforme se avance y se reflexione.

La transmisión del conocimiento en la universidad ubicua del futuro será con dispositivos tecnológicos como los hologramas estilo Stars Wars, el Instituto Tecnológico de Estudios Superiores de Monterrey (ITESM) ya inició con este tipo de experiencias, los profesores avatares se intensificarán, la enseñanza y el aprendizaje incorporado-embodied cognition se extenderá en todas los campos científicos, serán cotidianos los dispositivos cibernéticos incorporados al cuerpo y al cerebro que aumentarán las habilidades humanas de aprendizaje (como ejemplo, la antena del primer cyborg en el mundo), softwares conectados con la mente y el cuerpo humano serán una realidad normalizada. 
La universidad ubicua será el centro del conocimiento como un gran cerebro social que almacenará conocimiento y aprendizaje. No todas las universidades de la actualidad lograrán ser parte de este proceso. Habrá universidades que se queden en distintos momentos del cambio. Con un espíritu ni predictivo ni filosófico sino de deconstrucción del discurso dominante de la universidad empresarial, las universidades que se resistan al cambio quedarán en el olvido, universidades que transiten desde ahora hacia la investigación y la innovación como eje de su esencia darán pasos en la dirección de ser parte del futuro como algo incierto y no como algo determinado y esperado socialmente. Las universidades que potencialicen las capacidades científica y tecnológicas junto con sus capacidades sociales, culturales y artísticas estarán en mejores condiciones para adaptarse o transformarse con base en las exigencias del futuro que se puede leer más como una ficción que como una normativa social. La articulación de la universidad con la sociedad del siglo XXI será clave para la supervivencia de la universidad en su forma ubicua.

\section{Conclusión}

Los estudios del futuro de la universidad abren la posibilidad de pensar a la universidad de una forma distinta al discurso dominante del capitalismo académico. De analizar la universidad desde la prospectiva, previsión y la alteridad, y con ello contar con una visión más completa del futuro. Existe toda una gama de aportes conceptuales de los estudios del futuro que bien vale la pena aplicarlos en la comprensión y explicación del futuro de la universidad como una forma de prepararnos para la incertidumbre.

Abordar el futuro de la universidad no es algo nuevo, se ha hecho siempre, se ha realizado constantemente en una lógica lineal basada en la tendencia y en el manejo de variables que se conciben perpetuas. La narrativa dominante del futuro de la universidad nace, por lo general, en los organismos internacionales como el único futuro posible anulando la posibilidad de narrativas deseables del futuro de la universidad. Los actores universitarios tenemos que actuar en contra sentido de la meta prestablecida como único futuro. La constante ha sido que el futuro de la universidad está anclado en la economía, de una u otra forma, siempre en la economía.

Los estudios críticos del futuro con base en la epistemología de la deconstrucción nos colocan en la posibilidad de vislumbrar una nueva era de la universidad que deconstruya la narrativa dominante, y poder explorar narrativas alternativas que coloquen en el centro lo inédito y la incertidumbre. Un enfoque exploratorio nos coloca en la posibilidad de contestar la interrogante: ¿cómo será la universidad del futuro? La respuesta es que estamos en los umbrales de una nueva era de 
la universidad, la era de la universidad ubicua. La transmisión del conocimiento será por las redes neuronales conectadas entre sí de forma rizomática constituyéndose en el cerebro social de dimensiones de una galaxia, de la galaxia Gutenberg a la galaxia ubicua, donde la enseñanza-aprendizaje estarán fundidos en la conexión exploratoria de lo desconocido como reto de aumentar las capacidades humanas y no humanas en conjunto entre cyborg, robots y otras especies no humanas que se sumarán. Los implantes tecnológicos de conocimiento serán cosa común en los procesos de exploración entre lo desconocido y la incertidumbre.

La universidad ubicua estará centrada en la inteligencia híbrida, la comunicación científica estará pautada por la realidad aumentada y por hologramas conectados con la mente humana y con el cerebro cibernético. Los límites de los sentidos serán superados, acción que ya inició con el primer cyborg reconocido mundialmente. Que puede escuchar los colores; más delante, en la universidad ubicua, el oído humano podrá captar frecuencias ahora desconocidas, percibir olores con colores o sonidos. Hay todo un mundo por conquistar, los nuevos exploradores egresarán de la universidad ubicua.

En la nueva era de la universidad, la exploración no será territorial, será conocer lo que hay en el espacio donde ahora no conocemos ni percibimos nada, brotará una realidad que nos dejará sorprendidos, y, sobre todo, será superada nuestra ignorancia de las maravillas del silencio y del vacío. Esto está empezando, estamos a tiempo de acompañarnos en conjunto en la marcha del futuro de la universidad, hacia la universidad ubicua.

\section{Referencias}

Aoun, J. 2017. Higher Education in the Age of Artificial Intelligence. Londres: MIT Press.

Asimov, I. 2000. Sobre la ciencia ficción. Buenos Aires: Editorial Sudamericana.

Aubier, C. 2009. El gran libro de las artes adivinatorias. Santa Fe de Bogotá: Ediciones Robinbook.

British Council. 2015. Connecting Universities: Future models of higher education. Analysing innovation models for Afghanistan, Bangladesh, India Nepal, Pakistam and Sri Lanka. Londres: The Economist.

Chung, P. et al. 2012. Estudio prospectivo para la enseñanza superior virtual al 2030. Indistrial Data, 15(1). https://www.redalyc.org/pdf/816/81624969013. pdf (Consultado: 17 de julio, 2020).

Domínguez, J. 1972. Los objetivos y las etapas del proceso de planificación de la enseñanza superior. Revista de Educación Superior, vol. 1, México: ANUIES.

Eyring, H. y Christensen, C. 2011. The innovative University: Changing the DNA of higher eduaction, Washington, D.C.: American Council on Education. 
Fernández, A. 2005. Cuentos de ciencia ficción [Antología]. Buenos Aires: Estrada. Flechtheim, O. 1966. History and futurology. Meisenheim am Glan: Verlang Anton Hain.

Forward Thinking Platform. 2016. Glosario de términos utilizados en los Estudios de Futuro. https://www.gfar.net/sites/default/files/Forward\%20Thinking\%20 platform\%20Glossary_long\%20version_SP.pdf (Consultado: 18 de julio, 2020).

Glenn, J. et al. 2015. 2015-2016 Estado del Futuro. Washington, D.C.: The Millennium Project.

Gómez, D. 2019. El papel de los robots en la educación superior. Apel Asociación de proveedores de e-Learning. http://www.apel.es/papel-los-robots-la-educacion-del-futuro/ (Consultado: 14 de agosto, 2019).

Haraway, D. 1984. Manifiesto Ciborg. El sueño irónico de un lenguaje común para las mujeres en el circuito integrado. https://xenero.webs.uvigo.es/profesorado/beatriz_suarez/ciborg.pdf (Consultado: 18 de agosto, 2019).

Inayatullah, S. y Gidley, J. (2000). The university in transformation. Global perspectives on the future of the university. Wesport, CT.: Bergin \& Garvey.

Inayatullah, S. 2012a. Estudios del futuro: Teorías y metodologías. En Francisco González (comp.), Hay futuro. Visiones para un mundo mejor. Madrid: BBVA.

Inayatullah, S. 2012b. Malaysian universities in transformation. Journal of Futures Studies, 17(2). https://jfsdigital.org/articles-and-essays/2012-2/vol-17-no-2-december-2012/report/malaysian-universities-in-transformation/ (Consultado: 3 de agosto, 2019).

Laptev, G. 2016. The process of Russian educational reforms is underway. Russia Direct. From to University 1.0 to 4.0. Naturing innovation and entrepreneurship in Russian academia, 4(8).

López-Pellisa, T. 2018. Historia de la ciencia ficción en la cultura española. Madrid: La casa de la riqueza, Estudios de la Cultura de España.

López, V. 2017. e-Corpus iCorpus [Arte y tecnología]. Nuevos conceptos a las relaciones entre el cuerpo y la tecnología. Ciudad de México: Editorial Fontamara.

Manera L. 2020. STEAM and educational robotics: interdisciplinary approaches to robotics in early childhood and primary education. En Ferraguti F., Villani V., Sabattini L., Bonfè M. (eds.), Human-friendly robotics 2019. HFR 2019. Springer Proceedings in Advanced Robotics, vol 12. Springer, Cham. https://doi. org/10.1007/978-3-030-42026-0_8

Medina, J.1996. Los estudios del futuro y la prospectiva: Claves para la construcción social de las regiones. Serie Ensayos. ILPES: Santiago de Chile.

Medina, J. y Ortegón, E. 2006a. Manual de prospectiva y decisión estratégica: bases teóricas e instrumentos para América Latina. Santiago de Chile: ILPES.

Medina, J. 2006b. Visión compartida del futuro. Cali: Universidad del Valle. Monje, D. y Ortegón, N. 2016. Eugène Atgen, el cyborg de París y su relación doble 
vincular. ASRI Arte y Sociedad, Revista de Investigación, 11. Málaga: Universidad de Málaga.

Paracelso, T. 2001. Textos esenciales Paracelso. Madrid: Ediciones Siruela.

Pigem, J. 2018. Ángeles o robots. La interioridad humana en la sociedad hipertecnológica. Barcelona: Fragmentada Editorial.

Pulido, A. 2009. El futuro de la universidad. Un tema para el debate dentro y fuera de las universidades, Madrid: Delta Publicaciones Universitarias.

Ribeiro, F. y Lopes, G. (2020). Leraning robotics: a review. https://doi.org/10.1007/ s43154-020-00002-9. https://link.springer.com/content/pdf/10.1007/s43154020-00002-9.pdf (Consultado: 15 de julio, 2020).

Rivera, M. 1990. El futuro de las universidades en los países en desarrollo. Revista de Educación Superior, vol. 19. México: ANUIES.

Shuttleworth, M. 2009. Predicción en la investigación. https://explorable.com/es/ prediccion-en-la-investigacion (Consultado: 12 de julio, 2019).

Solano, J. 2001. Los estudios de futuro: Evalución y prospectiva. Revista Anales, 1(2). Caracas: Universidad Metropolitana.

Tapscott, D. y Tapscott, A. 2017. The Blockchain Revolution and Higher Education. EDUCASE Review. https://er.educase.edu/articles/2017/3/the-blockchain-revolition-and-higer-education (Consultado: 5 de agosto, 2019).

U.S. Department of Education. 2006. A test of leadership: Charting the future of U.S. Higher education. Washington, D.C.: U.S. Department of Education, Education Publications Center.

Varela, J. et al. 2020. Teaching STEM competencies through an educational mobile robot. En Zaphiris P., Ioannou A. (eds.), Learning and collaboration technologies. Human and technology ecosystems. HCII 2020. Lecture Notes in Computer Science, vol. 12206. Springer, Cham. https://doi.org/10.1007/978-3-03050506-6_38

Vergara, J. et al. 2010. Futurología: origen, evolución y métodos. Revista Palabra, 11. Cartagena de Indias: Universidad de Cartagena.

Yehya, N. 2013. Apuntes para una historia de la poshumanidad. Revista Letras Libres, https://www.letraslibres.com/mexico-espana/ciencia-y-tecnologia/apuntesuna-historia-la-poshumanidad (Consultado: 22 de agosto, 2019). 